\title{
Asymptotic Behavior for a Nonlocal Diffusion Equation in Domains with Holes
}

\author{
Carmen Cortázar, Manuel Elgueta, Fernando Quirós \& \\ NOEMÍ WOLANSKI
}

Communicated by P. RABINOWITZ

\begin{abstract}
The paper deals with the asymptotic behavior of solutions to a non-local diffusion equation, $u_{t}=J * u-u:=L u$, in an exterior domain, $\Omega$, which excludes one or several holes, and with zero Dirichlet data on $\mathbb{R}^{N} \backslash \Omega$. When the space dimension is three or more this behavior is given by a multiple of the fundamental solution of the heat equation away from the holes. On the other hand, if the solution is scaled according to its decay factor, close to the holes it behaves like a function that is $L$-harmonic, $L u=0$, in the exterior domain and vanishes in its complement. The height of such a function at infinity is determined through a matching procedure with the multiple of the fundamental solution of the heat equation representing the outer behavior. The inner and the outer behaviors can be presented in a unified way through a suitable global approximation.
\end{abstract}

\section{Introduction}

Let $\mathcal{H} \subset \mathbb{R}^{N}, N \geqq 3$, be a bounded open set with smooth boundary and let $\Omega=\mathbb{R}^{N} \backslash \mathcal{H}$. We do not assume $\mathcal{H}=\Omega^{c}$ to be connected, so it may represent one or several holes in an otherwise homogeneous medium. Our goal is to study the large-time behavior of the solution to the nonlocal heat equation in that exterior domain with zero data on the boundary, namely,

$$
\begin{cases}\partial_{t} u(x, t)=\int_{\mathbb{R}^{N}} J(x-y)(u(y, t)-u(x, t)) \mathrm{d} y & \text { in } \Omega \times(0, \infty), \\ u(x, t)=0 & \text { in } \mathcal{H} \times(0, \infty), \\ u(x, 0)=u_{0}(x) & \text { in } \mathbb{R}^{N},\end{cases}
$$

with a kernel $J$ that is assumed to be a nonnegative continuous function with unit integral. 
We will restrict ourselves to the case where $J$ is smooth, radially symmetric, with a compact support contained in the unit ball centered at the origin and $J(0)>0$. However, results similar to those we obtain here should hold for more general kernels.

The hypotheses on the initial data, $u_{0}$, are that they are nonnegative, bounded, integrable, and identically zero in the hole $\mathcal{H}$. We also assume, without loss of generality, that $0 \in \mathcal{H}$.

Evolution problems with this type of diffusion have been widely considered in the literature, since they can be used to model the dispersal of a species by taking into account long-range effects $[5,8,11]$. It has also been proposed as a model for phase transitions [3,4], and, quite recently, for image enhancement [12].

The nonlocal equation in (1) has been the subject of many recent works in the case where the spatial domain is $\mathbb{R}^{N}$, and also when it is a smooth bounded domain and is complemented by a suitable Dirichlet or Neumann type boundary condition, see the recent monograph [1] and the references therein. However, to our knowledge, this is the first time that an exterior domain has been considered.

When there are no holes, $\mathcal{H}=\emptyset$, mass is conserved, $\int_{\mathbb{R}^{N}} u(x, t) \mathrm{d} x=$ $\int_{\mathbb{R}^{N}} u_{0}(x) \mathrm{d} x$, and the solution to (1) behaves for large times as the solution $v$ to the local heat equation with diffusivity

$$
\alpha:=\frac{1}{2 N} \int_{\mathbb{R}^{N}}|z|^{2} J(z) \mathrm{d} z
$$

and the same initial data $[9,15]$. More precisely,

$$
\lim _{t \rightarrow \infty} t^{N / 2} \max _{x \in \mathbb{R}^{N}}|u(x, t)-v(x, t)|=0 .
$$

Hence the asymptotic behavior of $u$ can be described in terms of the fundamental solution of the heat equation with diffusivity $\alpha, \Gamma_{\alpha}$. This solution has a self-similar structure,

$$
\Gamma_{\alpha}(x, t)=t^{-N / 2} U_{\alpha}\left(\frac{x}{t^{1 / 2}}\right), \quad U_{\alpha}(y)=(4 \pi \alpha)^{-N / 2} \mathrm{e}^{-\frac{|y|^{2}}{4 \alpha}}
$$

Therefore, in self-similar variables we have convergence towards the stationary profile $M U_{\alpha}$, where $M=\int_{\mathbb{R}^{N}} u_{0}(x) \mathrm{d} x$, that is,

$$
\lim _{t \rightarrow \infty} \max _{y \in \mathbb{R}^{N}}\left|t^{N / 2} u\left(y t^{1 / 2}, t\right)-M U_{\alpha}(y)\right|=0 .
$$

Thus, there is an asymptotic symmetrization: whether the initial datum is radial or not, the large time behavior of $u$ is given by a radial profile, which, of course, has the same mass as the datum.

In the presence of holes the situation is very different. On one hand, mass is not conserved. On the other hand, the presence of the hole breaks (in general) the symmetry of the spatial domain, and an asymptotic symmetrization is no longer possible. 
Nevertheless, when $N \geqq 3$ we still have a conservation law, $\int_{\mathbb{R}^{N}} u(x, t) \phi(x) \mathrm{d} x=$ $\int_{\mathbb{R}^{N}} u_{0}(x) \phi(x) \mathrm{d} x$, with $\phi$ the unique solution to

$$
\begin{cases}J * \phi=\phi & \text { in } \Omega, \\ \phi=0 & \text { in } \mathcal{H}, \\ \phi(x) \rightarrow 1 & \text { as }|x| \rightarrow \infty\end{cases}
$$

Moreover, there is a non-trivial asymptotic mass which coincides with the conserved quantity $M^{*}=\int_{\mathbb{R}^{N}} u_{0}(x) \phi(x) \mathrm{d} x$. Besides, if we stand far away from the holes, they will be seen as just one point, and we may still expect some symmetrization. Indeed, we will prove that

$$
\lim _{t \rightarrow \infty} t^{N / 2} u\left(y t^{1 / 2}, t\right)=M^{*} U_{\alpha}(y) \quad \text { uniformly in }|y| \geqq \delta>0 .
$$

The only effect of the holes in this outer limit is the loss of mass. However, close to the holes, in the inner limit, their effects are much more important. On compact sets solutions still decay as $O\left(t^{-N / 2}\right)$. If we scale the solutions accordingly, we get that the new variable $w(x, t)=t^{N / 2} u(x, t)$ satisfies

$$
\partial_{t} w=J * w-w+\frac{N}{2} \frac{w}{t} .
$$

Thus, we expect $w$ to converge to an $L$-harmonic function $\Phi$ with zero 'boundary data',

$$
L \Phi:=J * \Phi-\Phi=0, \quad x \in \Omega, \quad \Phi=0, \quad x \in \mathcal{H} .
$$

This problem does not have uniqueness. However, a unique solution can be determined by prescribing the value at infinity. Indeed, if $\Phi \rightarrow C^{*}$ as $|x| \rightarrow \infty$, then $\Phi=C^{*} \phi$.

In order to select the right constant $C^{*}$ characterizing the asymptotic limit of $w$, we need some extra information. This will come from the outer limit, following a typical matched asymptotics procedure. Let us explain in some detail how this is done.

In view of the asymptotic behavior of $\phi$, we can describe the outer limit in the alternative form

$$
\lim _{t \rightarrow \infty} t^{N / 2} \sup \left\{\left|u(x, t)-M^{*} \phi(x) \Gamma_{\alpha}(x, t)\right|:|x| \geqq \delta \sqrt{t}\right\}=0 \quad \text { for all } \delta>0 .
$$

In addition, since $\lim _{t \rightarrow \infty} t^{N / 2} \Gamma_{\alpha}(x, t)=(4 \pi \alpha)^{-N / 2}$ uniformly on compact sets, the expected inner limit can be written as

$$
t^{N / 2}\left|u(x, t)-C^{*}(4 \pi \alpha)^{N / 2} \phi(x) \Gamma_{\alpha}(x, t)\right| \rightarrow 0 \text { as } t \rightarrow \infty
$$

uniformly in compact sets. We will prove that we can go beyond compact sets, and that this limit holds uniformly in a set of the form $|x| \leqq \bar{\delta} t^{1 / 2}$ for some $\bar{\delta}>0$. More precisely, there exists $\bar{\delta}>0$ such that

$$
\lim _{t \rightarrow \infty} t^{N / 2} \sup \left\{\left|u(x, t)-C^{*}(4 \pi \alpha)^{N / 2} \phi(x) \Gamma_{\alpha}(x, t)\right|:|x| \leqq \bar{\delta} \sqrt{t}, x \in \Omega\right\}=0 .
$$


Hence, the inner and the outer developments overlap, and they can be matched, leading to

$$
C^{*}=M^{*}(4 \pi \alpha)^{-N / 2} .
$$

Notice that we have been able to describe the inner and the outer behavior in a unified way. Hence we may gather the two results into a single theorem.

Theorem 1. Let $N \geqq 3, u_{0} \in L^{1}\left(\mathbb{R}^{N}\right) \cap L^{\infty}\left(\mathbb{R}^{N}\right), u_{0} \geqq 0$ and $M^{*}=$ $\int_{\mathbb{R}^{N}} u_{0}(x) \phi(x) \mathrm{d} x$. Then,

$$
t^{N / 2}\left|u(x, t)-M^{*} \phi(x) \Gamma_{\alpha}(x, t)\right| \rightarrow 0 \text { as } t \rightarrow \infty \text { uniformly in } \mathbb{R}^{N} .
$$

Remark 1. Let $\rho=\inf \{R: \operatorname{supp} J \subset B(0 ; R)\}$. The set $\Omega+B(0 ; \rho / 2)$ has only one unbounded component, $\mathcal{U}$. Though $\mathcal{U}$ may contain several components of $\Omega$, only one of them is really unbounded. Nevertheless, due to the non-local character of the diffusion operator, all the components of $\Omega$ contained in $\mathcal{U}$ are 'connected to infinity'. On the other hand, there may exist components of $\Omega$ which do not intersect $\mathcal{U}$, and which are, thus, 'truly' bounded. The function $\phi$ is identically 0 there. Therefore, the scaling we are using is not adequate to characterize the asymptotic behavior in such subdomains. Indeed, the decay rate in these kinds of bounded 'components' is exponential, and the asymptotic profile is an eigenfunction of the operator $L$ with zero Dirichlet boundary conditions in the complement of the 'component', associated to the first eigenvalue [9].

Remark 2. The asymptotic behavior for the case where there are no holes (4) can also be described in the form (6), since the solution to (5) when $\mathcal{H}=\emptyset$ is $\phi=1$.

When $N \leqq 2$, mass is expected to decay to zero (logarithmically when $N=2$, and like a power when $N=1)$, and the solutions will decay faster than $O\left(t^{-N / 2}\right)$. The analysis becomes much more involved, and is postponed to a future paper.

An analogous study in an exterior domain has been performed for the corresponding local problem in [13]. However, the author makes extensive use in the proofs of the explicit form of the fundamental solution of the local heat equation in the whole space. One of the main difficulties in the non-local case is that we do not have such an explicit expression.

An alternative approach, in which fundamental solutions do not play such a key role in the proofs, has been used for nonlinear (local problems), see for instance $[7,14]$. However, the operators under consideration in these cases are invariant under some scaling transformations, which is not the case in the present problem.

Nevertheless, although it will not be apparent from our proofs, scaling still plays a key role in the outer region. In fact, as observed in [10], under the usual parabolic scaling our operator 'converges' to $\alpha \Delta$. This is the reason the outer behavior is given by the fundamental solution to the heat equation with diffusivity $\alpha$. This idea has been used in [17] to study the asymptotic behavior for the nonlocal heat equation with absorption in a certain critical case.

We complete the study of the asymptotic behavior by analyzing the function $\phi$ at infinity. We prove that all the derivatives of $\phi$ behave at infinity as those of the fundamental solution of the Laplace operator. This result might be of independent interest. 
Notations. In what follows we will denote

$$
L u(x, t):=\int_{\mathbb{R}^{N}} J(x-y)(u(y, t)-u(x, t)) \mathrm{d} y .
$$

\section{Preliminaries}

On the notion of solution. Let $u_{0} \in L^{1}\left(\mathbb{R}^{N}\right), u_{0}=0$ almost everywhere in $\mathcal{H}$. A solution of (1) is a function $u \in C\left([0, \infty): L^{1}\left(\mathbb{R}^{N}\right)\right)$ such that for all $t>0$

$$
u(\cdot, t)=u_{0}+\int_{0}^{t}(J * u(\cdot, s)-u(\cdot, s)) \mathrm{d} s \text { a.e. in } \Omega, \quad u(\cdot, t)=0 \text { a.e. in } \mathcal{H} .(7)
$$

Subsolutions and supersolutions are defined as usual by replacing the equalities in the definition above by $\leqq$ or $\geqq$, respectively.

Remark 3. If $u$ is a solution, then $u \in L^{1}\left(\mathbb{R}^{N} \times[0, T]\right)$ for all $T>0$. Hence, (1) holds, not only in the sense of distributions, but also almost everywhere in $\Omega \times$ $(0, \infty)$. Moreover, we also have $u \in C^{1}\left([0, \infty): L^{1}\left(\mathbb{R}^{N}\right)\right)$, and the equation holds almost everywhere in $\Omega$ for all $t \geqq 0$.

Existence and uniqueness. Existence and uniqueness of a solution to (1) can be proved for any $u_{0} \in L^{1}\left(\mathbb{R}^{N}\right)$ such that $u_{0}=0$ almost everywhere in $\mathcal{H}$ in several ways, for example by means of semigroup theory. For the sake of completeness, we include a simple proof which uses a standard fixed point argument. We do not need to assume that $u_{0} \in L^{\infty}\left(\mathbb{R}^{N}\right)$ or restrict the sign.

Proposition 1. For any $u_{0} \in L^{1}\left(\mathbb{R}^{N}\right)$ such that $u_{0}=0$ almost everywhere in $\mathcal{H}$ there exists a unique solution of (1).

Proof. The proof is quite similar to the one for the case $\mathcal{H}=\emptyset$ performed in [9].

The set

$$
\mathcal{B}_{t_{0}}=\left\{u \in C\left(\left[0, t_{0}\right]: L^{1}\left(\mathbb{R}^{N}\right)\right): u(\cdot, t)=0 \text { a.e. in } \mathcal{H} \text { for all } t \in\left[0, t_{0}\right]\right\}
$$

endowed with the norm

$$
\|u \mid\|=\max _{0 \leqq t \leqq t_{0}}\|u(\cdot, t)\|_{L^{1}\left(\mathbb{R}^{N}\right)}
$$

is a Banach space. We define the operator $\mathcal{T}: \mathcal{B}_{t_{0}} \rightarrow \mathcal{B}_{t_{0}}$ through

$$
(\mathcal{T} u)(\cdot, t)= \begin{cases}u_{0}(\cdot)+\int_{0}^{t}(J * u(\cdot, s)-u(\cdot, s)) \mathrm{d} s & \text { a.e. in } \Omega \\ 0 & \text { a.e. in } \mathcal{H} .\end{cases}
$$

This operator turns out to be contractive if $t_{0}$ is small enough. Indeed,

$$
\begin{aligned}
\int_{\mathbb{R}^{N}} & |\mathcal{T} \varphi-\mathcal{T} \psi|(\cdot, t) \\
& \leqq \int_{\Omega} \int_{0}^{t}(|J *(\varphi-\psi)(\cdot, s)|+|\varphi-\psi|(\cdot, s)) \mathrm{d} s \\
& \leqq \int_{0}^{t}\left(\|J\|_{L^{1}\left(\mathbb{R}^{N}\right)}+1\right)\|(\varphi-\psi)(\cdot, s)\|_{L^{1}\left(\mathbb{R}^{N}\right)} \mathrm{d} s
\end{aligned}
$$


Hence, $\|\mathcal{T} \varphi-\mathcal{T} \psi\| \mid \leqq 2 t_{0}\|\varphi-\psi\|$. Thus, $\mathcal{T}$ is a contraction if $t_{0}<1 / 2$. Existence and uniqueness in the time interval $\left[0, t_{0}\right]$ now follow easily, using Banach's fixed point theorem. Since the length $t_{0}$ of the existence and uniqueness time interval does not depend on the initial data, we may iterate the argument to extend the result to all positive times.

Comparison. Comparison is an immediate consequence of the following $T$-contraction property in $L^{1}$. Again, we are assuming only that the initial data are integrable.

Theorem 2. Let $u_{1}, u_{2}$ be solutions of (1) having as initial data respectively $u_{1,0}, u_{2,0} \in L^{1}\left(\mathbb{R}^{N}\right)$. Then, for every $t \geqq 0$,

$$
\int_{\mathbb{R}^{N}}\left(u_{1}-u_{2}\right)_{+}(\cdot, t) \leqq \int_{\mathbb{R}^{N}}\left(u_{1,0}-u_{2,0}\right)_{+} .
$$

Proof. We subtract the equations for $u_{1}$ and $u_{2}$ and multiply by $\mathbb{1}_{\left\{u_{1}>u_{2}\right\}}$. Since $u_{1}-u_{2} \in C^{1}\left([0, \infty) ; L^{1}\left(\mathbb{R}^{N}\right)\right)$,

$$
\partial_{t}\left(u_{1}-u_{2}\right) \mathbb{1}_{\left\{u_{1}>u_{2}\right\}}=\partial_{t}\left(u_{1}-u_{2}\right)_{+} .
$$

On the other hand, just using that $0 \leqq \mathbb{1}_{\left\{u_{1}>u_{2}\right\}} \leqq 1$ and $J \geqq 0$ we get,

$$
J *\left(u_{1}-u_{2}\right) \mathbb{1}_{\left\{u_{1}>u_{2}\right\}} \leqq J *\left(u_{1}-u_{2}\right)_{+} .
$$

Finally, $\left(u_{1}-u_{2}\right) \mathbb{1}_{\left\{u_{1}>u_{2}\right\}}=\left(u_{1}-u_{2}\right)_{+}$. We end up with

$$
\partial_{t}\left(u_{1}-u_{2}\right)_{+} \leqq \begin{cases}J *\left(u_{1}-u_{2}\right)_{+}-\left(u_{1}-u_{2}\right)_{+} & \text {a.e. in } \Omega \\ 0 & \text { a.e. in } \mathcal{H}\end{cases}
$$

Integrating in space, and using Fubini's Theorem, we get

$$
\partial_{t} \int_{\mathbb{R}^{N}}\left(u_{1}-u_{2}\right)_{+}(\cdot, t) \leqq 0 .
$$

Remark 4. The same proof applies when there are no holes, $\Omega=\mathbb{R}^{N}$.

As a corollary we have, on one hand, comparison and, on the other hand, an $L^{1}$-contraction property for solutions,

$$
\left\|\left(u_{1}-u_{2}\right)(\cdot, t)\right\|_{L^{1}\left(\mathbb{R}^{N}\right)} \leqq\left\|u_{1,0}-u_{2,0}\right\|_{L^{1}\left(\mathbb{R}^{N}\right)} .
$$

The latter property implies the continuous dependence of solutions on the initial data, and a uniform control of the $L^{1}$-norm along time,

$$
\|u(\cdot, t)\|_{L^{1}\left(\mathbb{R}^{N}\right)} \leqq\left\|u_{0}\right\|_{L^{1}\left(\mathbb{R}^{N}\right)} .
$$

The proof of Theorem 2 applies under much weaker hypotheses, leading to a more general comparison principle that will be useful in the sequel. 
Theorem 3. Let $\{\Omega(t)\}_{t \geqq 0}$ be a continuous (with respect to the Hausdorff distance) family of open smooth domains of $\mathbb{R}^{N}$. Let $u_{1}$ and $u_{2}$ be two functions in the class $C^{1}\left([0, \infty) ; L^{1}\left(\mathbb{R}^{N}\right)\right)$ such that

$$
\partial_{t} u_{1}-L u_{1} \leqq 0, \partial_{t} u_{2}-L u_{2} \geqq 0 \text { for a.e. } x \in \Omega(t), \forall t>0,
$$

and $u_{1}(x, t) \leqq u_{2}(x, t)$ for almost everywhere $x \in \mathbb{R}^{N} \backslash \Omega(t), \forall t>0$. Then, for every $t \geqq 0$,

$$
\int_{\mathbb{R}^{N}}\left(u_{1}-u_{2}\right)_{+}(\cdot, t) \leqq \int_{\mathbb{R}^{N}}\left(u_{1}(x, 0)-u_{2}(x, 0)\right)_{+} .
$$

Remark 5. In order to compare a subsolution and a supersolution in $\Omega(t), t>$ 0 , we need them only to be ordered in $(\Omega(t)+B(0 ; 1)) \backslash \Omega(t), t>0$, and in $\Omega(0)+B(0 ; 1)$.

Time decay. Solutions to problem (1) satisfy

$$
u(\cdot, t)= \begin{cases}\mathrm{e}^{-t} u_{0}+\int_{0}^{t} \mathrm{e}^{-(t-s)} J * u(\cdot, s) \mathrm{d} s & \text { a.e. in } \mathbb{R}^{N} \backslash \mathcal{H} \\ 0 & \text { a.e. in } \mathcal{H} .\end{cases}
$$

Therefore, $u$ has the same spatial regularity as the initial datum $u_{0}$, but not more. In particular, if $u_{0}$ is not bounded, neither is $u(\cdot, t)$ for any later time.

On the contrary, if the initial datum is bounded, the solution will stay bounded as time goes by. Moreover, if $u_{0} \in L^{1}\left(\mathbb{R}^{N}\right) \cap L^{\infty}\left(\mathbb{R}^{N}\right)$, the solution decays like $O\left(t^{-N / 2}\right)$. To check this, we first observe that, since $u$ is a solution to (1) and $u(\cdot, t)=0$ almost everywhere in $\mathcal{H}$ for all $t \in[0, \infty)$, then

$$
u_{t}=L u-\mathcal{X}_{\mathcal{H}}(J * u) \text { in } \mathbb{R}^{N} \times(0, \infty) .
$$

Furthermore, if $u_{0} \geqq 0$, we have $u(x, t) \geqq 0$. Hence, the function $u$, which belongs to $C\left([0, \infty) ; L^{1}\left(\mathbb{R}^{N}\right)\right)$, is a subsolution to the Cauchy problem (no holes). Therefore, it lies below the solution $u_{\mathrm{C}}$ to the Cauchy problem with the same initial datum.

On the other hand, as we have already mentioned, see (4), if $u_{0} \in L^{1}\left(\mathbb{R}^{N}\right) \cap$ $L^{\infty}\left(\mathbb{R}^{N}\right)$, then $u_{\mathrm{C}}=O\left(t^{-N / 2}\right)$. Thus,

$$
0 \leqq u(x, t) \leqq C t^{-N / 2}
$$

Remark 6. In order to obtain the asymptotic behavior of $u_{\mathrm{C}}$, the paper [9] requires $u_{0}, \hat{u}_{0} \in L^{1}\left(\mathbb{R}^{N}\right)$. The paper [15] dispenses with the integrability assumption on $\hat{u}_{0}$, and on the sole hypothesis $u_{0} \in L^{1}\left(\mathbb{R}^{N}\right)$ proves that

$$
\lim _{t \rightarrow \infty} t^{N / 2} \max _{x \in \mathbb{R}^{N}}\left|u(x, t)-\mathrm{e}^{-t} u_{0}(x)-v(x, t)\right|=0,
$$

where $v$ has the same meaning as in (3). Therefore, the hypotheses $u_{0} \in L^{1}\left(\mathbb{R}^{N}\right) \cap$ $L^{\infty}\left(\mathbb{R}^{N}\right)$ are enough to have (4). 
$L^{\infty}$-solutions. Instead of the $L^{1}$-theory outlined above, an $L^{\infty}$-theory for (1) is also possible. Given an initial datum $u_{0} \in L^{\infty}\left(\mathbb{R}^{N}\right)$, an $L^{\infty}$-solution to problem (1) is a function $u \in C\left([0, \infty) ; L^{\infty}\left(\mathbb{R}^{N}\right)\right)$ such that (7) holds for all $t \geqq 0$.

As in the case of $L^{1}$-solutions, existence and uniqueness follow from a fixedpoint argument. Indeed,

$$
\mathcal{B}_{t_{0}}=\left\{u \in L^{\infty}\left(\mathbb{R}^{N} \times\left[0, t_{0}\right]\right): u(\cdot, t)=0 \text { a.e. in } \mathcal{H} \text { for all } t \in\left[0, t_{0}\right]\right\}
$$

endowed with the norm

$$
\|u\|=\max _{0 \leqq t \leqq t_{0}}\|u(\cdot, t)\|_{L^{\infty}\left(\mathbb{R}^{N}\right)}
$$

is a Banach space, and the operator $\mathcal{T}: \mathcal{B}_{t_{0}} \rightarrow \mathcal{B}_{t_{0}}$ defined by (8) satisfies $\| \mathcal{T} \varphi-$ $\mathcal{T} \psi\left\|\left|\leqq 2 t_{0}\|\varphi-\psi \mid\|\right.\right.$. Hence, it is contractive if $t_{0}<1 / 2$, which implies the local existence and uniqueness result. By iteration, taking as initial datum $u\left(\cdot, t_{0}\right) \in$ $L^{\infty}\left(\mathbb{R}^{N}\right)$, we obtain existence and uniqueness for $\left[0,2 t_{0}\right]$ and therefore for all times.

Besides, $L^{\infty}$-solutions depend continuously on the initial data. Indeed, let $u_{1}$ and $u_{2}$ be $L^{\infty}$-solutions with initial data $u_{1,0}, u_{2,0} \in L^{\infty}\left(\mathbb{R}^{N}\right)$. Since the functions $u_{i}$ satisfy (9) (with $u_{0}=u_{i, 0}$ ), an easy computation shows that for every $t>0$,

$$
\max _{s \in[0, t]}\left\|u_{1}(\cdot, s)-u_{2}(\cdot, s)\right\|_{L^{\infty}\left(\mathbb{R}^{N}\right)} \leqq\left\|u_{1,0}-u_{2,0}\right\|_{L^{\infty}\left(\mathbb{R}^{N}\right)} .
$$

In particular, the $L^{\infty}$-norm of a bounded solution does not increase with time,

$$
\|u(\cdot, t)\|_{L^{\infty}\left(\mathbb{R}^{N}\right)} \leqq\|u(\cdot, 0)\|_{L^{\infty}\left(\mathbb{R}^{N}\right)} \quad \forall t>0 .
$$

Essentially the same computation yields a comparison result for bounded suband supersolutions if the hole does not change with time. Let us examine this in some detail. Assume $u_{1,0} \leqq u_{2,0}$ in $\mathbb{R}^{N}$. Let $u_{1}$ be a bounded subsolution with initial datum $u_{1,0}$ and let $u_{2}$ be a bounded supersolution with initial datum $u_{2,0}$ in $\Omega=\mathbb{R}^{N} \backslash \mathcal{H}$ with $u_{1} \leqq u_{2}$ in $\mathcal{H}$ for every $t>0$. Let $u=u_{1}-u_{2}$ and $u_{0}=u_{1,0}-u_{2,0}$. Then,

$$
u(x, t) \leqq \begin{cases}\mathrm{e}^{-t} u_{0}(x)+\int_{0}^{t} \mathrm{e}^{-(t-s)} J * u(x, s) \mathrm{d} s & \text { if } x \in \Omega \\ 0 & \text { if } x \in \mathcal{H} .\end{cases}
$$

Hence, for $x \in \Omega$,

$$
u(x, t) \leqq \int_{0}^{t} \mathrm{e}^{-(t-s)} J * u(x, s) \mathrm{d} s \leqq \sup _{\mathbb{R}^{N} \times(0, t)}\left(1-\mathrm{e}^{-t}\right),
$$

with leads to

$$
\sup _{\mathbb{R}^{N} \times(0, t)} u \leqq \sup _{\mathbb{R}^{N} \times(0, t)} u\left(1-\mathrm{e}^{-t}\right) .
$$

Therefore, $\sup _{\mathbb{R}^{N} \times(0, t)} u \leqq 0$ for every $t>0$. This means that $u \leqq 0$. Thus, $u_{1} \leqq u_{2}$. 
Remark 7. The comparison result for bounded sub- and supersolutions of the Cauchy problem was proved in [16] using a different technique. The result of that paper is, in fact, more general, since it also applies to semilinear equations, as long as the nonlinearity is locally Lipschitz continuous. In addition, its proof copes without further ado with the case of holes that change with time.

An alternative proof of comparison for the case of one-dimensional exterior domains which do not change with time can be found in [2].

Remark 8. From now on we will always assume that $u_{0} \in L^{1}\left(\mathbb{R}^{N}\right) \cap L^{\infty}\left(\mathbb{R}^{N}\right)$ and $u_{0} \geqq 0$.

Representation formula. By (10), $u$ can be expressed in terms of the fundamental solution $F=F(x, t)$ to the operator $\partial_{t}-L$ in the whole space, studied in [9], by means of the variation of constants formula. Thus, for $t \geqq t_{0}$ we have

$$
\begin{aligned}
u(x, t)= & \int_{\mathbb{R}^{N}} F\left(x-y, t-t_{0}\right) u\left(y, t_{0}\right) \mathrm{d} y \\
& \quad-\int_{t_{0}}^{t} \int_{\mathbb{R}^{N}} F(x-y, t-s) \mathcal{X}_{\mathcal{H}}(y)(J * u(\cdot, s))(y) \mathrm{d} y \mathrm{~d} s .
\end{aligned}
$$

The fundamental solution can be decomposed as

$$
F(x, t)=\mathrm{e}^{-t} \delta(x)+\omega(x, t),
$$

where $\delta(x)$ is the Dirac mass at the origin in $\mathbb{R}^{N}$ and $\omega$ is defined via its Fourier transform as

$$
\hat{\omega}(\xi, t)=\mathrm{e}^{-t}\left(\mathrm{e}^{\hat{J}(\xi) t}-1\right)
$$

see [9], from where it is easy to see that $\omega$ is a smooth function and

$$
\int_{\mathbb{R}^{N}} \omega(x, t) \mathrm{d} x=\hat{\omega}(0, t)=1-\mathrm{e}^{-t}
$$

Combining (12) and (13), we obtain

$$
\begin{aligned}
u(x, t)= & \mathrm{e}^{-\left(t-t_{0}\right)} u\left(x, t_{0}\right)+\int_{\mathbb{R}^{N}} \omega\left(x-y, t-t_{0}\right) u\left(y, t_{0}\right) \mathrm{d} y \\
& -\int_{t_{0}}^{t} \mathrm{e}^{-(t-s)} \mathcal{X}_{\mathcal{H}}(x)(J * u(\cdot, s))(x) \mathrm{d} s \\
& -\int_{t_{0}}^{t} \int_{\mathbb{R}^{N}} \omega(x-y, t-s) \mathcal{X}_{\mathcal{H}}(y)(J * u(\cdot, s))(y) \mathrm{d} y \mathrm{~d} s .
\end{aligned}
$$

This formula will be the starting point to obtain both the behavior at infinity of $\phi$ and the outer large time behavior of $u$. 
Estimates for $\omega$. In order to profit from the representation formula (15), we need good estimates for the regular part, $\omega$, of the fundamental solution.

A first source of estimates comes from the asymptotic convergence of $\omega$ to the fundamental solution of the local heat equation with diffusivity $\alpha$, which is proved using Fourier transform techniques [15]. Indeed, given a multi-index $\beta=$ $\left(\beta_{1}, \ldots, \beta_{N}\right) \in \mathbb{N}_{0}^{N}$ of order $|\beta|=s$,

$$
t^{(N+s) / 2}\left\|D^{\beta} \omega(\cdot, t)-D^{\beta} \Gamma_{\alpha}(\cdot, t)\right\|_{L^{\infty}\left(\mathbb{R}^{N}\right)} \rightarrow 0 \text { as } t \rightarrow \infty,
$$

where $D^{\beta} f=\partial_{x_{1}}^{\beta_{1}} \cdots \partial_{x_{N}}^{\beta_{N}} f$. In particular,

$$
\left|D^{\beta} \omega(x, t)\right| \leqq C t^{-\frac{N+s}{2}} \text { if }|\beta|=s .
$$

These estimates give the right order of time decay. However, they do not take into account the spatial structure of $\omega$, and will not be enough for our purposes. We need to know something about the spatial decay of $\omega$ as $|x| \rightarrow \infty$.

A second source of estimates is the expansion

$$
\omega(x, t)=\mathrm{e}^{-t} \sum_{n=1}^{\infty} \frac{t^{n} J^{* n}(x)}{n !}, \quad J^{* n}=\underbrace{J * \cdots * J}_{n \text { times }},
$$

which follows by using the Taylor series of the exponential in (14). This expression, recently derived in [6], was used by the authors to obtain estimates giving the behavior of $\omega$ as $|x| \rightarrow \infty$ for each fixed $t$. However, though they give the right order of spatial decay for each time, they become very poor as $t \rightarrow \infty$.

Hence we need a different approach: we will obtain 'good' estimates through a comparison argument. Indeed, as observed in [17], $\omega$ is a solution to

$$
\begin{cases}\partial_{t} \omega(x, t)-L \omega(x, t)=\mathrm{e}^{-t} J(x) & \text { in } \mathbb{R}^{N} \times(0, \infty), \\ \omega(x, 0)=0 & \text { in } \mathbb{R}^{N},\end{cases}
$$

a fact that can be checked, for example, either by differentiating $\hat{\omega}(\xi, t)$ with respect to $t$ or by using that $\mathrm{e}^{-t} \delta(x)+\omega(x, t)$ is a solution of $\partial_{t} F-L F=0$. Good estimates will then follow from the use of appropriate barriers.

Proposition 2. The function $\omega$ is nonnegative. Moreover, given a multi-index $\beta \in \mathbb{N}_{0}^{N}$ of order $|\beta|=s$,

$$
\begin{gathered}
\left|D^{\beta} \omega(x, t)\right| \leqq C \frac{t}{|x|^{N+2+s}}, \\
\int_{\mathbb{R}^{N}}\left|D^{\beta} \omega(x, t)\right| \mathrm{d} x \leqq C t^{-s / 2} .
\end{gathered}
$$

The cases $s=0,1$ have already been proved in [17] through a clever use of the above mentioned comparison principle from [16]. The proof of the general case is completely analogous. The fact that $\omega$ is nonnegative was also proved in [17] and can also be seen from the series expansion (18).

Remark 9. Estimates (20) and (21) are in some sense optimal, since they are invariant under the 'parabolic' scaling $\left(\tau_{k} u\right)(x, t)=k^{N} u\left(k x, k^{2} t\right)$. This scaling will play a major part in some of our proofs. 


\section{The Stationary Problem}

As we have seen in the introduction, the $L$-harmonic function $\phi$ solving (5) plays a key role in the description of the asymptotic behavior of solutions to (1). In this section we prove that problem (5) is well posed, and we obtain some properties of $\phi$ that will be required later. Though not needed in the sequel, we complete our study of the stationary solution $\phi$ by precisely describing its behavior at infinity.

Remark on the notion of solution. In principle, we only need $\phi \in L_{\text {loc }}^{1}\left(\mathbb{R}^{N}\right)$, so that the convolution makes sense. But the equation implies that $\phi$ has the same regularity in $\bar{\Omega}$ as $J$ has in $\mathbb{R}^{N}$. Hence $\phi$ is at least continuous in $\Omega$ and, in our case, $\phi$ is smooth in $\Omega$. However, $\phi$ is not continuous across the boundary of $\Omega$.

\subsection{Existence and Uniqueness}

To prove existence we will approximate the domain $\Omega$ by a sequence of bounded domains, $\Omega_{n}=\Omega \cap B(0 ; n)$. Let us start by studying the Dirichlet problem for such bounded domains.

Lemma 1. Let $G \subset \mathbb{R}^{N}$ open and bounded.

(i) Given $f \in L^{\infty}\left(G^{c}\right)$, there exists a solution to problem

$$
L \phi(x)=0 \text { in } \bar{G}, \quad \phi=f(x) \text { in } \bar{G}^{c} .
$$

(ii) Let $\phi_{1}, \phi_{2} \in L^{\infty}\left(\mathbb{R}^{N}\right) \cap C(\bar{G})$ such that

$$
L \phi_{1} \geqq L \phi_{2} \text { in } \bar{G}, \quad \phi_{1} \leqq \phi_{2} \text { in } \bar{G}^{c} .
$$

Then $\phi_{1} \leqq \phi_{2}$ in $\mathbb{R}^{N}$.

Proof. Existence. Let $\mathcal{K}=\left\{\psi \in C(\bar{G}):\|\psi\|_{L^{\infty}(G)} \leqq\|f\|_{L^{\infty}\left(G^{c}\right)}\right\}$. We define an operator $\mathcal{T}: \mathcal{K} \rightarrow \mathcal{K}$ through the formula

$$
(\mathcal{T} \psi)(x)=\int_{G} J(x-y) \psi(y) \mathrm{d} y+\int_{G^{c}} J(x-y) f(y) \mathrm{d} y .
$$

Since $\|\mathcal{T} \psi\|_{L^{\infty}(\bar{G})} \leqq\|f\|_{L^{\infty}\left(G^{c}\right)}$ and $\|\mathcal{T} \psi\|_{C^{1}(\bar{G})} \leqq C_{J}\|f\|_{L^{\infty}\left(G^{c}\right)}$, we may apply Schauder's fixed point theorem to obtain that $\mathcal{T}$ has a fixed point $\hat{\phi} \in \mathcal{K}$. Then, the function

$$
\phi(x)= \begin{cases}\hat{\phi}(x), & x \in \bar{G}, \\ f(x), & x \in \bar{G}^{c},\end{cases}
$$

is the sought-after solution.

Comparison. Let $\phi=\phi_{1}-\phi_{2}$. Then,

$$
\phi(x) \leqq \int_{G} J(x-y) \phi(y) \mathrm{d} y .
$$


Assume for contradiction that $M=\max _{\bar{G}} \phi>0$. Let $x_{0} \in \bar{G}$ be a point at which the maximum is attained and let $G_{i}$ be the corresponding connected component. The set $K=\left\{x \in \bar{G}_{i}: \phi(x)=M\right\}$ is nonempty and closed. On the other hand,

$$
M=\phi\left(x_{0}\right) \leqq \int_{G} J\left(x_{0}-y\right) \phi(y) \mathrm{d} y \leqq M \int_{G} J\left(x_{0}-y\right) \mathrm{d} y \leqq M .
$$

This is possible only if $\phi(x)=M$ in $\bar{G} \cap \operatorname{supp} J\left(x_{0}-\cdot\right)$. Since $J(0)>0$, this implies that $\phi(x)=M$ in $\bar{G} \cap B\left(x_{0} ; \delta\right)$ for some $\delta>0$; hence $K$ is an open subset of $\bar{G}_{i}$. Since $\bar{G}_{i}$ is connected, we deduce that $K=\bar{G}_{i}$, so that, $\phi \equiv M>0$ in $\bar{G}_{i}$. But this implies that

$$
M=\phi\left(x_{0}\right) \leqq M \int_{G} J\left(x_{0}-y\right) \mathrm{d} y<M
$$

for any $x_{0} \in \partial G_{i}$ where the density of $\bar{G}_{i}^{c}$ is positive, a contradiction.

If in Lemma 1 we take $G=\Omega_{n}, f=0$ in $\mathcal{H}, f=1$ in $\Omega_{n}^{c} \backslash \mathcal{H}$, we obtain a monotone sequence $\left\{\phi_{n}\right\}_{n \in \mathbb{N}}$ converging to a function $\phi$. In order to prove that $\phi$ approaches 1 at infinity, we will compare the approximate solutions defined in bounded sets with a suitable barrier $z$. Hence we need to estimate $L z$.

Lemma 2. Let $z(x)=\left(|x|^{2}+b\right)^{-\gamma}, \gamma>0$. There exists a number $b=b(J, \gamma, N)>0$ such that

$$
L z(x) \leqq-2 \alpha \gamma\left(|x|^{2}+b\right)^{-(\gamma+1)}(N-2-2 \gamma),
$$

where $\alpha$ is the constant defined in (2).

Proof. After some cumbersome, but easy, computations, using Taylor's expansion and the radial symmetry of $J$, we find that

$$
\begin{aligned}
L z(x)= & -2 \alpha \gamma\left(|x|^{2}+b\right)^{-(\gamma+1)}(N-2-2 \gamma)-4 \alpha \gamma(\gamma+1)\left(|x|^{2}+b\right)^{-(\gamma+2)} b \\
& +O\left(\left(|x|^{2}+b\right)^{-(\gamma+2)}\right),
\end{aligned}
$$

from where the result follows just choosing $b$ large enough.

Proposition 3. There exists a unique solution to (5). Moreover, $0 \leqq \phi \leqq 1$ and there exists a positive constant $K$ such that

$$
1-\phi(x) \leqq \frac{K}{\left(|x|^{2}+1\right)^{(N-2) / 2}}, \quad x \in \Omega .
$$

Proof. Existence. Let $n_{0}$ in $\mathbb{N}$ be such that $\mathcal{H} \subset B\left(0 ; n_{0}\right)$. Consider the sequence of functions $\left\{\phi_{n}(x)\right\}_{n \geqq n_{0}}$, where $\phi_{n}$ is the solution to

$$
\begin{cases}L \phi_{n}(x)=0 & \text { in } B(0 ; n) \backslash \mathcal{H}, \\ \phi_{n}=0 & \text { in } \mathcal{H}, \\ \phi_{n}=1 & \text { in } B(0 ; n)^{c} .\end{cases}
$$


A simple comparison argument proves that $0 \leqq \phi_{n}(x) \leqq 1$ for all $n \geqq n_{0}, x \in$ $\mathbb{R}^{N}$. Hence, $\phi_{n}(x) \geqq \phi_{n+1}(x)$ in $(B(0 ; n))^{c}$, which yields, again by comparison, $0 \leqq \phi_{n+1}(x) \leqq \phi_{n}(x) \leqq 1$ for all $n \geqq n_{0}, x \in \mathbb{R}^{N}$. Therefore, the monotone limit

$$
\phi(x)=\lim _{n \rightarrow \infty} \phi_{n}(x)
$$

is well defined, and satisfies $0 \leqq \phi \leqq 1$. Letting $n \rightarrow \infty$ in (24), by the monotone convergence theorem we get

$$
L \phi(x)=0 \text { in } \Omega, \quad \phi=0 \text { in } \mathcal{H} .
$$

We still have to prove (23), which implies in particular that $\phi(x) \rightarrow 1$ as $|x| \rightarrow \infty$. This is done by comparison with the barrier $z$ described in Lemma 2 with $2 \gamma=N-2$. Indeed, by taking $K$ large enough, on one hand we have $K z \geqq 1=1-\phi_{n}$ in $\mathcal{H}, K z \geqq 1-\phi_{n}=0$ in $(B(0 ; n))^{c}$, and on the other hand $L(K z) \leqq 0=L\left(1-\phi_{n}\right)$ in $B(0 ; n) \backslash \mathcal{H}$. Hence, $K z \geqq 1-\phi_{n}$ in $\mathbb{R}^{N}$ and, passing to the limit, we obtain (23).

Uniqueness. Let $\phi$ and $\bar{\phi}$ be two solutions to (5). We consider the family of functions $\bar{\phi}_{\lambda}(x):=\bar{\phi}(x)+\lambda$. Since both $\phi$ and $\bar{\phi}$ are bounded, there is a value $\lambda_{1}$ such that $\bar{\phi}_{\lambda_{1}} \geqq \phi$ in $\mathbb{R}^{N}$. Let

$$
\lambda_{0}=\inf \left\{\lambda: \bar{\phi}_{\lambda} \geqq \phi \text { in } \mathbb{R}^{N}\right\} .
$$

We shall prove that $\lambda_{0}=0$, which means that $\bar{\phi} \geqq \phi$ in $\mathbb{R}^{N}$, from which uniqueness follows, since the roles of $\bar{\phi}$ and $\phi$ can be interchanged.

Assume for contradiction that $\lambda_{0}>0$. Since both $\phi(x)$ and $\bar{\phi}(x)$ tend to 1 as $|x| \rightarrow \infty$, there exists $R_{1}$ such that $\bar{\phi}_{\lambda_{0}}(x)>\phi(x)$ if $|x| \geqq R_{1}$. Moreover, $\bar{\phi}_{\lambda_{0}}>\phi$ in $\mathcal{H}$. Hence, as both $\bar{\phi}$ and $\phi$ are continuous in $R^{N} \backslash \mathcal{H}$, there exists $x_{0}$ in $\overline{B\left(0 ; R_{1}\right)} \backslash \mathcal{H}$ such that $\bar{\phi}_{\lambda_{0}}\left(x_{0}\right)=\phi\left(x_{0}\right)$. The set

$$
S=\left\{x \in \overline{B\left(0 ; R_{1}\right)} \backslash \mathcal{H}: \bar{\phi}_{\lambda_{0}}(x)=\phi(x)\right\}
$$

is closed and non-empty. If $x_{1}$ is a point in $\partial S$ where the density of $S^{c}$ is positive, then $\bar{\phi}_{\lambda_{0}}\left(x_{1}\right)=\phi\left(x_{1}\right)$ but $J * \bar{\phi}_{\lambda_{0}}\left(x_{1}\right)>J * \phi\left(x_{1}\right)$. Since this is a contradiction, we get that $\lambda_{0}=0$.

\subsection{Asymptotic Properties}

We now obtain the decay at infinity of the derivatives of $\phi$. In the sequel we let $\psi=1-\phi$.

Proposition 4. Given $s \in \mathbb{N}_{0}$, there exists a constant $C_{s}$ such that, for every multiindex $\beta \in \mathbb{N}_{0}^{N}$ of order $|\beta|=s$,

$$
\left|D^{\beta} \psi(x)\right| \leqq \frac{C_{s}}{|x|^{N-2+s}}, \quad x \in \Omega .
$$


Proof. The function $\phi$ is the unique bounded solution to the evolution problem (1) with initial data $\phi$. Hence, using the representation formula (15) with $t_{0}=0$ and $u\left(x, t_{0}\right)=\phi(x)$, we get,

$$
\begin{aligned}
\phi(x)= & \frac{1}{1-\mathrm{e}^{-t}} \int_{\mathbb{R}^{N}} \omega(x-y, t) \phi(y) \mathrm{d} y-\mathcal{X}_{\mathcal{H}}(x)(J * \phi)(x) \\
& -\frac{1}{1-\mathrm{e}^{-t}} \int_{0}^{t} \int_{\mathcal{H}} \omega(x-y, t-s)(J * \phi)(y) \mathrm{d} y \mathrm{~d} s .
\end{aligned}
$$

Therefore, since $\int_{\mathbb{R}^{N}} \omega(x, t) \mathrm{d} x=1-\mathrm{e}^{-t}$, we have

$$
\begin{aligned}
\psi(x)= & \frac{1}{1-\mathrm{e}^{-t}} \int_{\mathbb{R}^{N}} \omega(x-y, t) \psi(y) \mathrm{d} y+\mathcal{X}_{\mathcal{H}}(x)(J * \phi)(x) \\
& +\frac{1}{1-\mathrm{e}^{-t}} \int_{0}^{t} \int_{\mathcal{H}} \omega(x-y, t-s)(J * \phi)(y) \mathrm{d} y \mathrm{~d} s .
\end{aligned}
$$

Given $k>0$, we denote

$$
\begin{array}{ll}
\phi^{k}(x)=k^{N-2} \phi(k x), & \psi^{k}(x)=k^{N-2} \psi(k x), \\
J_{k}(x)=k^{N} J(k x), & \omega_{k}(x, t)=k^{N}\left(k x, k^{2} t\right),
\end{array}
$$

and $\mathcal{H}_{k}=\left\{x \in \mathbb{R}^{N}: k x \in \mathcal{H}\right\}$. If we scale equation (26) according to these recipes, we get

$$
\begin{aligned}
\psi^{k}(x)= & \frac{1}{1-\mathrm{e}^{-t}} \int_{\mathbb{R}^{N}} \omega_{k}\left(x-y, k^{-2} t\right) \psi^{k}(y) \mathrm{d} y+\mathcal{X}_{\mathcal{H}_{k}}(x)\left(J_{k} * \phi^{k}\right)(x) \\
& +\frac{1}{1-\mathrm{e}^{-t}} \int_{0}^{t} \int_{\mathcal{H}_{k}} \omega_{k}\left(x-y, k^{-2}(t-s)\right)\left(J_{k} * \phi^{k}\right)(y) \mathrm{d} y \mathrm{~d} s .
\end{aligned}
$$

Let $\delta>0$ be given. There exists a value $k_{\delta}$ such that $\mathcal{X}_{\mathcal{H}_{k}}(x)=0$ if $|x| \geqq \delta / 2$ and $k \geqq k_{\delta}$. Therefore,

$$
\begin{aligned}
D^{\beta} \psi^{k}(x)= & \underbrace{\frac{1}{1-\mathrm{e}^{-t}} \int_{\mathbb{R}^{N}} D^{\beta} \omega_{k}\left(x-y, k^{-2} t\right) \psi^{k}(y) \mathrm{d} y}_{\mathcal{A}} \\
& +\underbrace{\frac{1}{1-\mathrm{e}^{-t}} \int_{0}^{t} \int_{\mathcal{H}_{k}} D^{\beta} \omega_{k}\left(x-y, k^{-2}(t-s)\right)\left(J_{k} * \phi^{k}\right)(y) \mathrm{d} y \mathrm{~d} s}_{\mathcal{B}},
\end{aligned}
$$

if $|x| \geqq \delta / 2$ and $k \geqq k_{\delta}$.

We start by estimating $\mathcal{B}$. If $y \in \mathcal{H}_{k}$ and $|x| \geqq \delta$, then $|x-y| \geqq \delta / 2$ if $k \geqq k_{\delta}$. On the other hand, since $0 \leqq \phi^{k}(x) \leqq \bar{k}^{N-2}$, we have, in addition, $0 \leqq J_{k} * \phi^{k} \leqq k^{N-2}$. So, recalling that $\left|\mathcal{H}_{k}\right|=\bar{C} k^{-N}$ and the bounds in (20)_ which are scaling invariant-we get

$$
\begin{aligned}
|\mathcal{B}| & \leqq \frac{k^{N-2}}{1-\mathrm{e}^{-t}} \int_{0}^{t} \int_{\mathcal{H}_{k}} \frac{C k^{-2}(t-s)}{|x-y|^{N+s+2}} \mathrm{~d} y \mathrm{~d} s \\
& \leqq C_{\delta, N} \frac{k^{-4}}{1-\mathrm{e}^{-t}} \int_{0}^{t}(t-s) \mathrm{d} s=C_{\delta, N} \frac{k^{-4}}{1-\mathrm{e}^{-t}} t^{2} \leqq C_{\delta, N} \quad \text { if } \quad t=k^{2} .
\end{aligned}
$$


Now we estimate $\mathcal{A}$ with $t=k^{2}$. Recall that $0 \leqq \psi(x) \leqq C /|x|^{N-2}$. Therefore we have, again using (20) and (21),

$$
\begin{aligned}
|\mathcal{A}| \leqq & \frac{1}{1-\mathrm{e}^{-k^{2}}} \int_{|y|<\delta / 2}\left|D^{\beta} \omega_{k}(x-y, 1)\right| \frac{C}{|y|^{N-2}} \mathrm{~d} y \\
& \quad+\frac{C_{\delta, N}}{1-\mathrm{e}^{-k^{2}}} \int_{|y| \geqq \delta / 2}\left|D^{\beta} \omega_{k}(x-y, 1)\right| \mathrm{d} y \\
\leqq & \frac{C_{\delta, N}}{1-\mathrm{e}^{-k^{2}}} \int_{|y|<\delta / 2} \frac{\mathrm{d} y}{|y|^{N-2}}+\frac{C_{\delta, N}}{1-\mathrm{e}^{-k^{2}}} \leqq C_{\delta, N} .
\end{aligned}
$$

In conclusion, given $\delta>0$ there exists $k_{\delta}$ such that, if $k \geqq k_{\delta}$ and $|x| \geqq \delta$, then $\left|D^{\beta} \psi^{k}(x)\right| \leqq C_{\delta, N}$.

Let us take $|x|=1, C_{1}=C_{1, N}, y=k x$ so that $k=|y|$. Then, we get

$$
|y|^{N-2+s}\left|D^{\beta} \psi(y)\right|=k^{N-2+s}\left|D^{\beta} \psi(k x)\right|=\left|D^{\beta} \psi^{k}(x)\right| \leqq C_{1} \quad \text { if }|y|=k \geqq k_{1} .
$$

This proves estimate (25), except for a bounded set. However, $\phi$ is smooth in $\Omega$, and hence the estimate is obviously true for bounded sets.

Remark 10. The proof of the bound (25) only requires $J \in C^{s+1}$.

Though the second derivatives of $\psi$ decay like $O\left(|x|^{-N}\right)$ as $|x| \rightarrow \infty$, a special combination, the Laplacian, decays faster. In particular, $\Delta \psi$ is integrable at infinity.

Corollary 1. There exists a constant $C$ such that $|\Delta \psi(x)| \leqq \frac{C}{|x|^{N+2}}$ in $\Omega$.

Proof. Using Taylor's expansion we get

$$
L \psi(x)=\alpha \Delta \psi(x)+O\left(\max _{|\beta|=4}\left\|D^{\beta} \psi\right\|_{L^{\infty}\left(B_{1}(x)\right)}\right) .
$$

Since $\psi$ is $L$-harmonic, the result follows from the estimate (25) for the fourth order derivatives.

Remark 11. The proof of Corollary 1 only requires $J \in C^{4}$. If we weaken this assumption and only require $J \in C^{3}$, we get $|\Delta \psi(x)| \leqq C /|x|^{N+1}$, and the Laplacian of $\psi$ is still integrable at infinity.

We can profit from the integrability of $\Delta \psi$ at infinity to obtain the precise asymptotic behavior of $\psi$.

Proposition 5. There exists a constant $C^{*}$ such that for every multi-index $\beta \in \mathbb{N}_{0}^{N}$ of order $|\beta|=s$,

$$
|x|^{N-2+s}\left|D^{\beta} \psi(x)-D^{\beta} \psi^{*}(x)\right| \rightarrow 0 \text { as }|x| \rightarrow \infty, \quad \psi^{*}(x)=C^{*}|x|^{2-N} .
$$


Proof. Scaling and compactness. From (25) we get that the scaled functions $\psi^{k}$ satisfy $\left|D^{\beta} \psi^{k}(x)\right| \leqq C /|x|^{N-2+s}$ if $|\beta|=s$. Hence, thanks to Arzelà-Ascoli's theorem, for any sequence $\left\{k_{n}\right\}_{n \in \mathbb{N}}$ there exist a subsequence, that we name again $\left\{k_{n}\right\}_{n \in \mathbb{N}}$, and a function $\psi^{*}$ such that, for any multi-index $\beta$,

$$
D^{\beta} \psi^{k_{n}} \rightarrow D^{\beta} \psi^{*} \quad \text { uniformly on compact sets of } \mathbb{R}^{N} \backslash\{0\} .
$$

Moreover, $\psi^{*}$ inherits from the functions $\psi^{k}$ the estimate

$$
0 \leqq \psi^{*}(x) \leqq C /|x|^{N-2} .
$$

Identification of the limit along subsequences. We consider the scaled operator $L_{k}$ defined by $L_{k} v=k^{2}\left(J_{k} * v-v\right)$. Performing a Taylor expansion, it is easy to check that $L_{k} \varphi \rightarrow \alpha \Delta \varphi$ uniformly for any $\varphi \in C_{\mathrm{c}}^{\infty}\left(\mathbb{R}^{N}\right)$.

Now let $\varphi \in C_{\mathrm{c}}^{\infty}\left(\mathbb{R}^{N} \backslash\{0\}\right)$. We write

$$
\int_{\mathbb{R}^{N}} \psi^{*} \alpha \Delta \varphi=\int_{\mathbb{R}^{N}} \psi^{k} L_{k} \varphi+\int_{\mathbb{R}^{N}}\left(\psi^{*}-\psi^{k}\right) \alpha \Delta \varphi+\int_{\mathbb{R}^{N}} \psi^{k}\left(\alpha \Delta \varphi-L_{k} \varphi\right) .
$$

Since $L_{k} \psi^{k}=0$, we have $\int_{\mathbb{R}^{N}} \psi^{k} L_{k} \varphi=\int_{\mathbb{R}^{N}} L_{k} \psi^{k} \varphi=0$. Besides, using (25), as $k \rightarrow \infty$ we have

$$
\left|\int_{\mathbb{R}^{N}} \psi^{k}\left(\alpha \Delta \varphi-L_{k} \varphi\right)\right| \leqq C \int_{\mathbb{R}^{N} \cap\left(\operatorname{supp} \varphi+B_{1 / k}(0)\right)}\left|\alpha \Delta \varphi-L_{k} \varphi\right| \rightarrow 0 .
$$

Thus, taking $k=k_{n}$ in (30) and letting $n \rightarrow \infty$, we get $\int_{\mathbb{R}^{N}} \psi^{*} \alpha \Delta \varphi=0$, which implies that $\Delta \psi^{*}=0$ in $\mathbb{R}^{N} \backslash\{0\}$. Since $|x|^{N-2} \psi^{*}$ is bounded, see estimate (29), we may conclude that there exists a constant $C^{*}$ such that $\psi^{*}(x)=C^{*} /|x|^{N-2}$. Uniqueness of the limit. We next prove that the constant $C^{*}$ is independent of the sequence $\left\{k_{n}\right\}_{n \in \mathbb{N}}$.

A direct computation shows that

$$
\int_{\partial B(0 ; R)} \frac{\partial \psi^{*}}{\partial r} \mathrm{~d} S=C^{*}(2-N) N V_{N} \text { for all } R>0,
$$

where $V_{N}$ stands for the volume of the unit ball in $\mathbb{R}^{N}$. On the other hand, if $\mathcal{H} \subset \subset B_{R_{0}}$,

$$
\int_{\partial B(0 ; R)} \frac{\partial \psi}{\partial r} \mathrm{~d} S=\int_{\partial B\left(0 ; R_{0}\right)} \frac{\partial \psi}{\partial r} \mathrm{~d} S+\int_{B_{R} \backslash B_{R_{0}}} \Delta \psi(x) \mathrm{d} x \text { for all } R>R_{0} .
$$

Since $\int_{B_{R} \backslash B_{R_{0}}} \Delta \psi(x) \mathrm{d} x$ has a finite limit as $R \rightarrow \infty$, see Corollary 1 , $\int_{\partial B(0 ; R)} \frac{\partial \psi}{\partial r} \mathrm{~d} S$ also has a limit, that we denote by $\mu$.

Let $y=k x$. On one hand, we have $|y|^{N-1} \nabla \psi(y)=|x|^{N-1} \nabla \psi^{k}(x)$ and on the other, $|y|^{N-1} \nabla \psi^{*}(y)=|x|^{N-1} \nabla \psi^{*}(x)$. Therefore, taking $k=k_{n}$ and $|x|=1$, which implies $|y|=k_{n}$, and using (28), we get

$$
|y|^{N-1}\left|\nabla \psi(y)-\nabla \psi^{*}(y)\right|=\left|\nabla \psi^{k_{n}}(x)-\nabla \psi^{*}(x)\right|<\varepsilon
$$


if $|y|=k_{n}$ and $n$ is large. Hence,

$$
\left|\int_{\partial B\left(0 ; k_{n}\right)} \frac{\partial \psi}{\partial r} \mathrm{~d} S-C^{*}(2-N) N V_{N}\right|=\left|\int_{\partial B\left(0 ; k_{n}\right)}\left(\frac{\partial \psi}{\partial r}-\frac{\partial \psi^{*}}{\partial r}\right) \mathrm{d} S\right|<C \varepsilon
$$

if $n$ is large. We conclude that, $\left.C^{*}=\mu\left((2-N) N V_{N}\right)\right)^{-1}$.

Rephrasing the limit. Take $y=k x$ and $|x|=1$. In the above steps we have proved that

$$
|y|^{N-2+s} D^{\beta} \psi(y)=k^{N-2+s} D^{\beta} \psi(k x)=D^{\beta} \psi^{k}(x) \rightarrow D^{\beta} \psi^{*}(x)
$$

as $|y|=k \rightarrow \infty$. Since $D^{\beta} \psi^{*}(x)=|y|^{N-2+|\beta|} D^{\beta} \psi^{*}(y)$ (remember that $\left.|x|=1\right)$, this is just (27).

Remark 12. To get (27) it is enough to have $J \in C^{\max (3, s)}$. We need at least $J \in C^{3}$, no matter the order of the derivative we are considering, in order for $\Delta \psi$ to be integrable at infinity, which is one of the main ingredients of the proof.

\section{Conservation Law and Asymptotic Mass}

We start our study of the evolutionary problem (1) with a conservation law that will be of the highest importance for establishing the behavior of the solution in the far field.

Proposition 6. Let $u$ be the solution to (1) and $\phi$ the solution to (5). Then,

$$
\int_{\mathbb{R}^{N}} u(x, t) \phi(x) \mathrm{d} x=\int_{\mathbb{R}^{N}} u_{0}(x) \phi(x) \mathrm{d} x:=M^{*} \text { for every } t>0 .
$$

Proof. Since $u(x, t)=\phi(x)=0$ for $x \in \mathcal{H}$, we have

$$
\begin{aligned}
\frac{d}{d t} \int_{\mathbb{R}^{N}} u(x, t) \phi(x) \mathrm{d} x & =\int_{\mathbb{R}^{N}} u_{t}(x, t) \phi(x) \mathrm{d} x \\
& =\int_{\mathbb{R}^{N}} \int_{\mathbb{R}^{N}} J(x-y)(u(y, t)-u(x, t)) \phi(x) \mathrm{d} y \mathrm{~d} x \\
& =\int_{\mathbb{R}^{N}} \int_{\mathbb{R}^{N}} J(x-y)(\phi(y)-\phi(x)) u(x, t) \mathrm{d} y \mathrm{~d} x \\
& =0 .
\end{aligned}
$$

Though mass is not conserved, there is a non-trivial asymptotic mass, which coincides with the conserved quantity $M^{*}$.

Proposition 7. Let $u$ be the solution to $(1)$ and $M^{*}=\int_{\mathbb{R}^{N}} u_{0}(x) \phi(x) \mathrm{d} x$. The mass of the solution at time $t, M(t)=\int_{\mathbb{R}^{N}} u(x, t) \mathrm{d} x$, satisfies $M(t) \rightarrow M^{*}$ as $t \rightarrow \infty$. 
Proof. In fact,

$$
\begin{aligned}
\left|\int_{\mathbb{R}^{N}} u(x, t) \mathrm{d} x-M^{*}\right| & =\int_{\mathbb{R}^{N}} u(x, t)(1-\phi(x)) \mathrm{d} x \\
& \leqq \int_{|x|<\eta \sqrt{t}} u(x, t) \mathrm{d} x+\int_{|x| \geqq \eta \sqrt{t}} u(x, t)(1-\phi(x)) \mathrm{d} x \\
& \leqq C t^{-N / 2}(\eta \sqrt{t})^{N}+C \frac{1}{(\eta \sqrt{t})^{N-2}} \int_{\mathbb{R}^{N}} u(x, t) \mathrm{d} x \\
& \leqq C \eta^{N}+C\left\|u_{0}\right\|_{L^{1}\left(\mathbb{R}^{N}\right)} \frac{1}{(\eta \sqrt{t})^{N-2}} .
\end{aligned}
$$

Hence, choosing $\eta$ so that $C \eta^{N}<\varepsilon$ and taking lim $\sup _{t \rightarrow \infty}$ we get,

$$
\limsup _{t \rightarrow \infty}\left|\int_{\mathbb{R}^{N}} u(x, t) \mathrm{d} x-M^{*}\right| \leqq \varepsilon .
$$

We conclude by letting $\varepsilon \rightarrow 0$.

\section{Far-Field Limit}

In this section we study the large time behavior of the solution to (1) in sets of the form $\left\{|x|^{2} \geqq \delta t\right\}$, with $\delta>0$.

Theorem 4. Let $u$ be the solution of (1) and $M^{*}$ the asymptotic mass given in (31). Then, for every $\delta>0$,

$$
t^{N / 2}\left\|u(x, t)-M^{*} \Gamma_{\alpha}(x, t)\right\|_{L^{\infty}\left(\left\{|x|^{2} \geqq \delta t\right\}\right)} \rightarrow 0 \text { as } t \rightarrow \infty .
$$

Proof. Let $t_{0}>0$ and $t \geqq 2 t_{0}$. From the representation formula (15) we have

$$
\begin{aligned}
& t^{N / 2}\left|u(x, t)-M^{*} \Gamma_{\alpha}(x, t)\right| \leqq \underbrace{t^{N / 2} \mathrm{e}^{-\left(t-t_{0}\right)} u\left(x, t_{0}\right)}_{I_{1}} \\
& +\underbrace{t^{N / 2}\left|\int_{\mathbb{R}^{N}} \omega\left(x-y, t-t_{0}\right) u\left(y, t_{0}\right) \mathrm{d} y-M^{*} \Gamma_{\alpha}(x, t)\right|}_{I_{2}} \\
& +\underbrace{t^{N / 2} \int_{t_{0}}^{t} \mathrm{e}^{-(t-s)} \mathcal{X}_{\mathcal{H}}(x)(J * u(\cdot, s))(x) \mathrm{d} s}_{I_{3}} \\
& +\underbrace{t^{N / 2} \int_{t_{0}}^{t} \int_{\mathbb{R}^{N}} \omega(x-y, t-s) \mathcal{X}_{\mathcal{H}}(y)(J * u(\cdot, s))(y) \mathrm{d} y \mathrm{~d} s}_{I_{4}} .
\end{aligned}
$$

Let $\delta>0$ and $|x|^{2} \geqq \delta t$. We have the following estimates for the quantities $I_{i}$ : 
- $I_{1} \leqq C t^{N / 2} \mathrm{e}^{-t / 2} \rightarrow 0$ as $t \rightarrow \infty$.

- The estimate for $I_{2}$ is the most involved, and is split into three parts,

$$
\begin{aligned}
I_{2} \leqq & \underbrace{t^{N / 2} \int_{\mathbb{R}^{N}}\left|\omega\left(x-y, t-t_{0}\right)-\Gamma_{\alpha}\left(x-y, t-t_{0}\right)\right| u\left(y, t_{0}\right) \mathrm{d} y}_{I_{2,1}} \\
& +\underbrace{t^{N / 2}\left|\int_{\mathbb{R}^{N}} \Gamma_{\alpha}\left(x-y, t-t_{0}\right) u\left(y, t_{0}\right) \mathrm{d} y-M\left(t_{0}\right) \Gamma_{\alpha}(x, t)\right|}_{I_{2,2}} \\
& +\underbrace{t^{N / 2} \Gamma_{\alpha}(x, t)\left|M\left(t_{0}\right)-M^{*}\right|}_{I_{2,3}} .
\end{aligned}
$$

- Using (16),

$$
\begin{aligned}
I_{2,1} & \leqq t^{N / 2}\left\|u\left(\cdot, t_{0}\right)\right\|_{L^{1}\left(\mathbb{R}^{N}\right)}\left\|\omega\left(\cdot, t-t_{0}\right)-\Gamma_{\alpha}\left(\cdot, t-t_{0}\right)\right\|_{L^{\infty}\left(\mathbb{R}^{N}\right)} \\
& =t^{N / 2}\left\|u_{0}\right\|_{L^{1}\left(\mathbb{R}^{N}\right)} o\left(\left(t-t_{0}\right)^{-\frac{N}{2}}\right) .
\end{aligned}
$$

Thus, $\lim _{t \rightarrow \infty} I_{2,1}=0$.

- The well-known asymptotics for solutions of the local heat equation imply $\lim _{t \rightarrow \infty} I_{2,2}=0$.

- By Proposition 7, if $t_{0}$ is large enough, $I_{2,3} \leqq C_{\alpha}\left|M\left(t_{0}\right)-M^{*}\right|<\varepsilon$.

- For $t_{0}$ large enough, $x \notin \mathcal{H}$. Hence, $I_{3}=0$.

- If $t_{0}$ is large enough and $y \in \mathcal{H},|x-y| \geqq|x| / 2$. On the other hand, by (11) $0 \leqq u(x, s) \leqq C s^{-N / 2}$ and therefore, $0 \leqq(J * u(\cdot, s))(x) \leqq C s^{-N / 2}$. Thus, using the space-time bound (20) for $\omega$,

$$
\begin{aligned}
I_{4} & \leqq C t^{N / 2} \int_{t_{0}}^{t} \int_{\mathcal{H}} \frac{t-s}{|x-y|^{N+2}} s^{-N / 2} \mathrm{~d} y \mathrm{~d} s \leqq C\left(\frac{t}{|x|^{2}}\right)^{\frac{N}{2}+1} t_{0}^{-\frac{N}{2}+1} \\
& \leqq C \delta^{-\frac{N}{2}-1} t_{0}^{-\frac{N}{2}+1}<\varepsilon
\end{aligned}
$$

if $t_{0}$ is large enough.

Gathering all the above estimates, we get

$$
\limsup _{t \rightarrow \infty}\left\|t^{N / 2} u(x, t)-t^{N / 2} M^{*} \Gamma_{\alpha}(x, t)\right\|_{L^{\infty}\left(|x|^{2} \geqq \delta t\right)} \leqq 2 \varepsilon .
$$

We finally let $\varepsilon \rightarrow 0$.

As a corollary of the outer behavior, we can do better in the proof of Proposition 7 to obtain the rate of decay of the mass to its asymptotic limit.

Corollary 2. Under the assumptions of Proposition 7, we have

$$
M(t)=M^{*}+K t^{-\frac{N-2}{2}}+o\left(t^{-\frac{N-2}{2}}\right),
$$

where $K=C^{*} M^{*} \int_{\mathbb{R}^{N}} U_{\alpha}(\xi)|\xi|^{2-N} \mathrm{~d} \xi, C^{*}=\lim _{|x| \rightarrow \infty}(1-\phi(x))|x|^{N-2}$. 
Proof. We have from the conservation law (31) that

$$
t^{\frac{N-2}{2}}\left(M(t)-M^{*}\right)=t^{\frac{N-2}{2}} \int_{\mathbb{R}^{N}} u(x, t)(1-\phi(x)) \mathrm{d} x .
$$

In order to estimate this last integral, we split it into two parts,

$$
\begin{aligned}
t^{\frac{N-2}{2}} & \int_{\mathbb{R}^{N}} u(x, t)(1-\phi(x)) \mathrm{d} x \\
= & \underbrace{t^{\frac{N-2}{2}} \int_{\left\{|x| \leqq \delta t^{1 / 2}\right\}} u(x, t)(1-\phi(x)) \mathrm{d} x}_{I_{1}}+\underbrace{t^{\frac{N-2}{2}} \int_{\left\{|x| \geqq \delta t^{1 / 2}\right\}} u(x, t)(1-\phi(x)) \mathrm{d} x}_{I_{2}}
\end{aligned}
$$

for $\delta>0$ and $t$ big enough. Using the estimate $0 \leqq 1-\phi(x) \leqq c_{1}|x|^{2-N}$ and the bound $0 \leqq u(x, t) \leqq C t^{-N / 2}$ we get $0 \leqq I_{1} \leqq C \delta^{2}$. For $I_{2}$, we perform the change of variables $x=\xi t^{1 / 2}$, and get

$$
\begin{aligned}
\lim _{t \rightarrow \infty} I_{2} & =\int_{\{|\xi| \geqq \delta\}} \lim _{t \rightarrow \infty} \frac{|\xi|^{N-2}\left(1-\phi\left(\xi t^{1 / 2}\right)\right) t^{\frac{N-2}{2}}}{|\xi|^{N-2}} u\left(\xi t^{1 / 2}, t\right) t^{N / 2} \mathrm{~d} \xi \\
& =C^{*} M^{*} \int_{\{|\xi| \geqq \delta\}} U_{\alpha}(\xi)|\xi|^{2-N} \mathrm{~d} \xi .
\end{aligned}
$$

Hence

$$
\begin{aligned}
& C^{*} M^{*} \int_{\{|\xi| \geqq \delta\}} U_{\alpha}(\xi)|\xi|^{2-N} \mathrm{~d} \xi \leqq \liminf _{t \rightarrow \infty} t^{\frac{N-2}{2}}\left(M(t)-M^{*}\right) \\
& \quad \leqq \limsup _{t \rightarrow \infty} t^{\frac{N-2}{2}}\left(M(t)-M^{*}\right) \leqq C \delta^{2}+C_{*} M^{*} \int_{\{|\xi| \geqq \delta\}} U_{\alpha}(\xi)|\xi|^{2-N} \mathrm{~d} \xi
\end{aligned}
$$

from where the result follows just by letting $\delta \rightarrow 0$.

Remark 13. The amount of mass, $M_{L}(u)$, lost in the evolution is given by

$$
M_{L}(u):=\int_{\mathbb{R}^{N}} u_{0}(x) \mathrm{d} x-\lim _{t \rightarrow \infty} \int_{\mathbb{R}^{N}} u(x, t) \mathrm{d} x=\int_{\mathbb{R}^{N}}(1-\phi(x)) u_{0}(x) \mathrm{d} x>0 .
$$

Therefore, the influence of the hole structure is felt at the asymptotic level through the projection of the initial data onto $\psi:=1-\phi$, which represents in this way the dissipation capacity of $\mathcal{H}$.

Notice that $\psi$ is the $L$-harmonic function defined in $\Omega$ that takes a value of 1 on $\mathcal{H}$ and of 0 at infinity. Hence, by analogy with the standard (local) potential theory, we may say that $\psi$ is the function that measures the $L$-capacity of $\mathcal{H}$, by means of the formula

$$
\operatorname{cap}(\mathcal{H})=\inf _{\{u \geqq 1 \text { on } \mathcal{H}\}} \int_{\Omega} \int_{\Omega} J(x-y)(u(x)-u(y))^{2} \mathrm{~d} x \mathrm{~d} y .
$$




\section{Near-Field Limit}

In view of Theorem 4, what is left to complete the proof of Theorem 1 is to show that the limit (6) is uniformly valid in sets of the form $\left\{|x|^{2}<\delta t\right\}$ for some $\delta>0$. This is the goal of this section.

Theorem 5. Let $u$ be the solution to (1). There exists a value $\delta>0$ such that

$$
\lim _{t \rightarrow \infty} t^{N / 2}\left\|u(x, t)-M^{*} \phi(x) \Gamma_{\alpha}(x, t)\right\|_{L^{\infty}\left(\left\{|x|^{2}<\delta t\right\}\right)}=0 .
$$

Instead of (34), we will prove

$$
\lim _{t \rightarrow \infty} t^{N / 2}\left\|u(x, t)-M^{*} \phi(x) \omega(x, t)\right\|_{L^{\infty}\left(\left\{|x|^{2}<\delta t\right\}\right)}=0,
$$

which turns out to be equivalent, thanks to (16) with $|\beta|=0$. Notice also that, using again (16), together with the asymptotic behavior of $\phi$, we may rewrite (32) in a similar way,

$$
\lim _{t \rightarrow \infty} t^{N / 2}\left\|u(x, t)-M^{*} \phi(x) \omega(x, t)\right\|_{L^{\infty}\left(\left\{|x|^{2} \geqq \delta t\right\}\right)}=0 .
$$

In order to prove (35) we will construct suitable barriers approaching the asymptotic limit as $t \rightarrow \infty$. We choose $\kappa \in(0,1), \gamma \in(0,(N-2-\kappa) / 2)$, and then define, for any $K_{ \pm}>0$,

$$
v_{ \pm}(x, t)=\phi(x) \omega(x, t) \pm K_{ \pm} t^{-\frac{N+\kappa}{2}} z(x),
$$

with $z$ as in Lemma 2, $z(x)=\left(|x|^{2}+b\right)^{-\gamma}$. We take $b$ large enough, so that (22) is satisfied.

Lemma 3. Let $R>0$ and $v_{ \pm}$as above. There exists a value $\delta_{*}>0$, independent of $K_{ \pm}$, such that for all $\delta \in\left(0, \delta^{*}\right), K_{ \pm} \geqq 1$, we have

$$
\partial_{t} v_{+}-L v_{+} \geqq 0, \quad \partial_{t} v_{-}-L v_{-} \leqq 0, \quad R^{2} \leqq|x|^{2} \leqq \delta t .
$$

Proof. On one hand,

$$
\partial_{t} v_{+}(x, t)=\phi(x) \partial_{t} \omega(x, t)-\frac{N+\kappa}{2 t} K_{+} t^{-\frac{N+\kappa}{2}} z(x) .
$$

On the other hand, using that $\phi$ is $L$-harmonic and (22), we get

$$
\begin{gathered}
L v_{+}(x, t) \leqq \phi(x) L \omega(x, t)+\int_{\mathbb{R}^{N}} J(x-y)(\phi(y)-\phi(x))(\omega(y, t)-\omega(x, t)) \mathrm{d} y \\
-\frac{2 \alpha \gamma(N-2-2 \gamma)}{|x|^{2}+b} K_{+} t^{-\frac{N+\kappa}{2}} z(x) .
\end{gathered}
$$

Hence, since $\omega$ solves (19) and $\phi \geqq 0$,

$$
\begin{aligned}
\partial_{t} v_{+}-L v_{+} \geqq & -\underbrace{\int_{\mathbb{R}^{N}} J(x-y)|\phi(y)-\phi(x)||\omega(y, t)-\omega(x, t)| \mathrm{d} y}_{\mathcal{A}} \\
& +K_{+} t^{-\frac{N+\kappa}{2}} z(x) \underbrace{\left(\frac{2 \alpha \gamma(N-2-2 \gamma)}{|x|^{2}+b}-\frac{N+\kappa}{2 t}\right)}_{\mathcal{B}} .
\end{aligned}
$$


Estimate for $\mathcal{A}$. We first notice that there is a constant $C>0$ such that

$$
|\phi(x)-\phi(y)| \leqq \frac{C}{|x|^{N-1}} \quad \text { if }|x-y| \leqq 1 .
$$

Indeed, let $\hat{R}$ be such that $\mathcal{H} \subset B(0 ; \hat{R})$. Since $|\phi(x)-\phi(y)| \leqq 1$, inequality (38) is obviously true for $|x| \leqq \max \{2, \hat{R}+1\}$ with $C=(\max \{2, \hat{R}+1\})^{N-1}$. On the other hand, if $|x|>\max \{2, \hat{R}+1\}$, Taylor's expansion yields

$$
|\phi(x)-\phi(y)| \leqq \max _{|\beta|=1} \max _{\xi \in B(x ; 1)}\left|D^{\beta} \phi(\xi)\right| \quad \text { if }|x-y| \leqq 1 .
$$

The result now follows from the estimate (25) with $|\beta|=1$, since $|\xi| \geqq|x| / 2$ for all $\xi \in B(x ; 1)$ whenever $|x| \geqq 2$.

Combining (39) with (17), we conclude that there is a constant $C>0$ such that

$$
\mathcal{A} \leqq \frac{C t^{-\frac{N+1}{2}}}{|x|^{N-1}}=\frac{C t^{-\frac{N+\kappa}{2}}\left(|x|^{2} / t\right)^{\frac{1-\kappa}{2}}}{|x|^{N-\kappa}} \leqq \frac{C t^{-\frac{N+\kappa}{2}} \delta^{\frac{1-\kappa}{2}}}{|x|^{N-\kappa}} \quad \text { if }|x|^{2} \leqq \delta t .
$$

Estimate for $\mathcal{B}$. If $R^{2} \leqq|x|^{2} \leqq \delta t$ and $\delta \leqq \frac{\alpha \gamma(N-2-2 \gamma) R^{2}}{(N+\kappa)\left(R^{2}+b\right)}$, we have

$$
\begin{aligned}
\frac{N+\kappa}{2 t} & \leqq \frac{\delta(N+\kappa)}{|x|^{2}}=\frac{\delta(N+\kappa)\left(R^{2}+b\right)}{R^{2}|x|^{2}+b|x|^{2}} \\
& \leqq \frac{\delta(N+\kappa)\left(R^{2}+b\right)}{R^{2}\left(|x|^{2}+b\right)} \leqq \frac{\alpha \gamma(N-2-2 \gamma)}{|x|^{2}+b} .
\end{aligned}
$$

Thus, with this choice of $\delta$,

$$
\mathcal{B} \geqq \frac{\alpha \gamma(N-2-2 \gamma)}{|x|^{2}+b} .
$$

The above estimates for $\mathcal{A}$ and $\mathcal{B}$ yield, if $K_{+} \geqq 1$,

$$
\partial_{t} v_{+}-L v_{+} \geqq \frac{t^{-\frac{N+\kappa}{2}}}{\left(|x|^{2}+b\right)^{\gamma+1}}\left(\alpha \gamma(N-2-2 \gamma)-\frac{\left.C \delta^{\frac{1-\kappa}{2}}\left(|x|^{2}+b\right)^{\gamma+1}\right)}{|x|^{N-\kappa}}\right) .
$$

On the other hand, since $2(\gamma+1) \leqq N-\kappa$,

$$
\frac{\left(|x|^{2}+b\right)^{\gamma+1}}{|x|^{N-\kappa}} \leqq \frac{\left(R^{2}+b\right)^{\gamma+1}}{R^{N-\kappa}} .
$$

Therefore, taking $\delta$ small enough, we get

$$
\partial_{t} v_{+}-L v_{+} \geqq 0, \quad R^{2} \leqq|x|^{2} \leqq \delta t
$$

for all $K_{+} \geqq 1$. Notice that the threshold value of $\delta$ does not depend on $K_{+}$.

An analogous argument leads to the statement concerning $v_{-}$, since

$$
\mathrm{e}^{-t} J(x) \leqq t^{\frac{N+1}{2}}|x|^{1-N} \quad \text { if } R^{2} \leqq|x|^{2} \leqq \delta t
$$

whenever $\|J\|_{\infty} \leqq R^{2} / \delta^{\frac{N+1}{2}}$. 
Proof of Theorem 5. Let $R>0$ such that $B(0 ; R) \subset \mathcal{H}$. As a consequence of Lemma 3, we know that there is a value $\delta>0$ such that

$$
\partial_{t} v_{+}-L v_{+} \geqq 0, \quad x \in \Omega \cap\left\{R^{2}<|x|^{2} \leqq \delta t\right\}, t>R^{2} / \delta .
$$

Let now $\varepsilon>0$. By (36), (16) with $|\beta|=0$, and the asymptotic behavior of $\phi$, there exists a time $t_{0}>0$ such that $u(x, t) \leqq(1+\varepsilon) M^{*} \phi(x) \omega(x, t)$ if $(\delta t)^{1 / 2} \leqq|x| \leqq$ $(\delta t)^{1 / 2}+1, t \geqq t_{0}$. Hence, for any $K_{+} \geqq 0$,

$$
u(x, t) \leqq(1+\varepsilon) M^{*} v_{+}(x, t) \text { if }(\delta t)^{1 / 2} \leqq|x| \leqq(\delta t)^{1 / 2}+1, t \geqq t_{0} .
$$

On the other hand, it is obvious that there exists a constant $K_{+} \geqq 1$ such that $u\left(x, t_{0}\right) \leqq(1+\varepsilon) M^{*} v_{+}\left(x, t_{0}\right)$ if $|x| \leqq\left(\delta t_{0}\right)^{1 / 2}+1$. Finally, we also have that $u(x, t)=0 \leqq(1+\varepsilon) M^{*} v_{+}(x, t), x \in \mathcal{H}, t \geqq t_{0}$. Therefore, by comparison, see Theorem 3 and Remark 5,

$$
u(x, t) \leqq(1+\varepsilon) M^{*} v_{+}(x, t), \quad|x| \leqq(\delta t)^{1 / 2}, t \geqq t_{0} .
$$

Hence, using the decay estimate (17), if $|x| \leqq(\delta t)^{1 / 2}, t \geqq t_{0}$, we have

$$
\begin{aligned}
t^{N / 2}(u(x, t) & \left.-M^{*} \phi(x) \omega(x, t)\right) \\
& \leqq \varepsilon M^{*} \phi(x) t^{N / 2} \omega(x, t)+(1+\varepsilon) M^{*} K_{+} t^{-\frac{\kappa}{2}} z(x) \\
& \leqq \varepsilon M^{*} C+(1+\varepsilon) M^{*} K_{+} t^{-\frac{\kappa}{2}} b^{-\gamma} .
\end{aligned}
$$

Letting $t \rightarrow \infty$ and then $\varepsilon \rightarrow 0$, we conclude that

$$
\limsup _{t \rightarrow \infty} t^{N / 2}\left(u(x, t)-M^{*} \phi(x) \omega(x, t)\right) \leqq 0 .
$$

An analogous argument shows that

$$
(1-\varepsilon) M^{*} v_{-}(x, t) \leqq u(x, t), \quad|x| \leqq(\delta t)^{1 / 2}, t \geqq t_{0} .
$$

Hence, $\liminf _{t \rightarrow \infty} t^{N / 2}\left(u(x, t)-M^{*} \phi(x) \omega(x, t)\right) \geqq 0$.

As a corollary, we obtain the behavior on compact sets.

Corollary 3. Let $u$ be the solution to (1). Then,

$$
t^{N / 2} u(x, t) \rightarrow \frac{M^{*}}{(4 \pi \alpha)^{N / 2}} \phi(x) \text { uniformly in compact subsets of } \mathbb{R}^{N} .
$$

Remark 14. The limit (40) holds uniformly in $\Omega \cap\left\{|x|^{2} \leqq \delta(t) t\right\}$, as long as $\lim _{t \rightarrow \infty} \delta(t)=0$.

Acknowledgments All authors supported by FONDECYT grants 7090027 and 1110074 . The third author supported by the Spanish Project MTM2008-06326-C02-01. The fourth author supported by the Argentine Council of Research, CONICET under the project PIP625, Res. $845 / 10$ and UBACYT X117. N. Wolanski is a member of CONICET. 


\section{References}

1. Andreu, F., Mazón, J.M., Rossi, J.D., Toledo, J.: Nonlocal Diffusion Problems. Mathematical Surveys and Monographs, 165. American Mathematical Society, Providence; Real Sociedad Matemática Española, Madrid, 2010

2. Bates, P.W., Chen, F.: Spectral analysis of traveling waves for nonlocal evolution equations. SIAM J. Math. Anal. 38(1), 116-126 (2006)

3. Bates, P.W., ChmaJ, A.: An integrodifferential model for phase transitions: stationary solutions in higher dimensions. J. Stat. Phys. 95(5-6), 1119-1139 (1999)

4. Bates, P.W., Chmaj, A.: A discrete convolution model for phase transitions. Arch. Rational Mech. Anal. 150(4), 281-305 (1999)

5. Bates, P.W., Zhao, G.: Existence, uniqueness and stability of the stationary solution to a nonlocal evolution equation arising in population dispersal. J. Math. Anal. Appl. 332(1), 428-440 (2007)

6. Brändle, C., Chasseigne, E., Ferreira, R.: Unbounded solutions of the nonlocal heat equation. Commun. Pure Appl. Anal. 10(6), 1663-1686 (2011)

7. Brändle, C., Quirós, F., VÁzquez, J.L.: Asymptotic behaviour of the porous media equation in domains with holes. Interfaces Free Bound. 9(2), 211-232 (2007)

8. Carrillo, C., Fife, P.: Spatial effects in discrete generation population models. J. Math. Biol. 50(2), 161-188 (2005)

9. Chasseigne, E., Chaves, M., Rossi, J.D.: Asymptotic behavior for nonlocal diffusion equations. J. Math. Pures Appl. (9) 86(3), 271-291 (2006)

10. Cortázar, C., Elgueta, M., Rossi, J.D., Wolanski, N.: How to approximate the heat equation with Neumann boundary conditions by nonlocal diffusion problems. Arch. Rational Mech. Anal. 187(1), 137-156 (2008)

11. FIFE, P.: Some nonclassical trends in parabolic and parabolic-like evolutions. Trends in Nonlinear Analysis, 153-191. Springer, Berlin, 2003

12. Gilboa, G., Osher, S.: Nonlocal operators with application to image processing. Multiscale Model. Simul. 7(3), 1005-1028 (2008)

13. Herraiz, L.A.: A nonlinear parabolic problem in an exterior domain. J. Differ. Equ. 142(2), 371-412 (1998)

14. IAgar, R.G., VÁzQuez, J.L.: Asymptotic analysis of the $p$-Laplacian flow in an exterior domain. Ann. Inst. H. Poincaré Anal. Non Linéaire 26(2), 497-520 (2009)

15. Ignat, L.I., Rossi, J.D.: Refined asymptotic expansions for nonlocal diffusion equations. J. Evol. Equ. 8(4), 617-629 (2008)

16. Lederman, C., Wolanski, N.: Singular perturbation in a nonlocal diffusion model. Commun. Partial Differ. Equ. 31(1-3), 195-241 (2006)

17. Terra, J., WolansKi, N.: Large time behavior for a nonlocal diffusion equation with absorption and bounded initial data. Discrete Contin. Dyn. Syst. 31(2), 581-605 (2011)

Departamento de Matemática, Pontificia Universidad Católica de Chile, Casilla 306, Correo 22, Santiago, Chile. e-mail: ccortaza@mat.puc.cl

and

Departamento de Matemática, Pontificia Universidad Católica de Chile, Casilla 306, Correo 22, Santiago, Chile. e-mail: melgueta@mat.puc.cl 
Nonlocal Diffusion in Domains with Holes

Departamento de Matemáticas, Universidad Autónoma de Madrid, 28049 Madrid, Spain.

e-mail: fernando.quiros@uam.es and

Departamento de Matemática, FCEyN, UBA, and IMAS, CONICET, 1428 Buenos Aires, Argentina. e-mail:wolanski@dm.uba.ar

(Received November 10, 2011 / Accepted April 2, 2012)

(C) Springer-Verlag (2012) 